\title{
Expectation bubbles in a spin model of markets: Intermittency from frustration across scales
}

\author{
Stefan Bornholdt \\ Institut für Theoretische Physik, Universität Kiel, Leibnizstrasse 15, D-24098 Kiel, Germany, \\ bornholdt@theo-physik.uni-kiel.de
}

\begin{abstract}
A simple spin model is studied, motivated by the dynamics of traders in a market where expectation bubbles and crashes occur. The dynamics is governed by interactions which are frustrated across different scales: While ferromagnetic couplings connect each spin to its local neighborhood, an additional coupling relates each spin to the global magnetization. This new coupling is allowed to be anti-ferromagnetic. The resulting frustration causes a metastable dynamics with intermittency and phases of chaotic dynamics. The model reproduces main observations of real economic markets as power-law distributed returns and clustered volatility.
\end{abstract}

Keywords: econophysics, dynamics of markets, statistical mechanics of spin models, order-disorder transitions, metastability, self-organization.

To be published in Int. J. Mod. Phys. C 12 (2001), No 5.

Market dynamics emerging from a large number of interacting agents have raised considerable interest [1]. The resulting phenomena often are difficult to capture in simple models, in particular equilibrium concepts fail in describing specific dynamical properties of markets. Among such phenomena are expectation bubbles and subsequent crashes of expectation driven quantities $[2,4$.

Recently, fruitful attempts have been made to understand such emergent phenomena in systems of many interacting agents, e.g. [5]. Remarkably simple models can in principle capture essential features and, vice versa, provide toy systems that are as such interesting for statistical physics (but would hardly be written down from a pure physics perspective). One example of a simple model is the El Farol bar problem [8], most commonly known in a simplified formulation as the minority game [9]. In this game, agents split into two groups, however, only being in the minority group is rewarded and thus beneficial for each agent. This leads to a globally frustrated state as every single agent will try to reach this state. Similar interactions occur in real markets as, for example, it is often desirable to be in the minority when buying or selling a certain commodity. The minority game describes the evolution of strategic choices in a collective system within such a simple frustrated boundary condition. Beyond this aspect, the elementary minority game does not provide a model for the more detailed dynamics of financial markets, e.g. the dynamics of prices, as it is a very simplistic model. A recent attempt to include more general aspects of market dynamics into the minority game shows that quite complicated extensions are necessary, at the price of dropping the elegant simplicity of the original minority game 10$]$.

A complementary class of dynamical models for markets is formed by recent models of stock markets where trading agents are simulated, including an explicit price formation process, e.g. [7, 11, 12]. A particularly realistic model is the one by Lux and Marchesi [12,13] who classify the agents into two basic strategies ("fundamentalists" and "optimistic/pessimistic chartists"). Prices form as a result of the trading activity, and strategies are chosen by each agent on the basis of profitability w.r.t. past actions. The resulting model reproduces several non-trivial properties observed in real markets, as power-law distributed returns ret $(t)=\ln (p(t))-\ln (p(t-1))$ of prices $p(t)$, and a high autocorrelation of price volatility. Also, on the level of strategies, it shows the phenomenon that the number of chartists (or noise traders) correlates with phases of high volatility, as also seen in real markets. The Lux-Marchesi-Model therefore is quite successful. From the theoretical point of view its only drawback is a high complexity with more than 10 free parameters and considerable tuning. While this does not matter in economic applications of the model, a theoretical analysis of the basic mechanisms at work is not easy, facing a large number of details in the model.

In this article, therefore, a third path will be sketched, formulating a model with maximum simplicity, while including details of strategic interactions in a market. It can be viewed as an extremely simplified version of a stock market model in the form of a spin model with only two alternatives, designed to simulate the dynamics of expectations in systems of many agents. While spin models have a tradition in economic theory 114,17 , the critical properties of such models are often difficult to relate to real economic situations, at least without tuning. In particular, large fluctuations as often seen in real economic data 18.19] usually appear only near the critical point of spin models. One possibility to capture these features in a spin model is to reformulate a stock market model in terms of a generalized spin models as, for example, demonstrated by Chowdhury and Stauffer who recast the Cont-Bouchaud-Model in terms of "super spins" 20]. Also the random field Ising model can be related to critical properties of stock market dynamics [21]. 
Let us here take a different approach and rather start from scratch by asking, what are the basic forces at work? There are at least two major conflicting forces seen in economic action:

1. "Do what your neighbors do", as often seen in the action of noise traders and as modeled in the herd behavior of strategic choices in the Lux-Marchesi-Model, and:

2. "Do what the minority does", as often followed by traders with knowledge about fundamental values and as modeled in the minority game.

We here combine these two conflicting interactions in a simple spin model: On the one hand, the neighbor interaction is represented as a standard nearest neighbor interaction of a spin model. On the other hand, a coupling to the minority as a global observable is introduced by a coupling to the global magnetization of the spin system.

Consider a model with $i=1, \ldots, N$ spins with orientations $S_{i}(t)= \pm 1$. The dynamics of the spins depends on the local field $h_{i}(t)$, for simplicity assume that each spin is updated with a heat-bath dynamics according to

$$
\begin{aligned}
& S_{i}(t+1)=+1 \quad \text { with } \quad p=1 /\left[1+\exp \left(-2 \beta h_{i}(t)\right)\right] \\
& S_{i}(t+1)=-1 \quad \text { with } \quad 1-p
\end{aligned}
$$

The local field containing the interactions discussed above is further specified by

$$
h_{i}(t)=\sum_{j=1}^{N} J_{i j} S_{j}-\alpha C_{i}(t) \frac{1}{N} \sum_{j=1}^{N} S_{j}(t) .
$$

The first term is chosen as a local Ising Hamiltonian with nearest neighbor interactions $J_{i j}=J$ and $J_{i j}=0$ for all other pairs. This term thus induces local ferromagnetic order. The second term is a global coupling to the magnetization of the system with a coupling $\alpha>0$. Its sign determines the strategy of agent $i$. It is specified separately by a second spin $C_{i}(t)$, representing the strategy of agent $i$ with respect to the magnetization. In particular, this second coupling allows for the case of spins frustrated across scales, seeking ferromagnetic order locally, but anti-ferromagnetic order globally. Similar couplings to the total magnetization are known for magnets with dipole interactions as "demagnetizing field" which in the context of market models are reminiscent of the welfare state mode of the Levy-Solomon-Huang-Model [22].

What is the basic dynamics of this system? Several scenarios can be realized depending on value and dynamics of the strategy spin field. Let us consider the simplest possibilities. First, consider the case where the strategy is always $C_{i}(t)=1, \quad \forall i, t$. Each agent then, besides the local ferromagnetic coupling to the neighbors, has an anti-ferromagnetic coupling to the magnetization. This, in a sense follows a motivation reminiscent of the minority game, inducing a force to align with the minority of spins in the system. In contrast to the minority game, however, it is complemented by a conflicting ferromagnetic term which the minority game lacks. The dynamics with $C_{i}(t)=1$ then corresponds to traders, who, in addition to a basic level of ferromagnetic noise trading, have a desire to join the global minority, for example in order to invest in possible future gains. Thus the $C_{i}(t)=1$ traders can be called fundamentalists. If every agent follows this strategy, the global dynamics of the model will quickly lead to a near-vanishing magnetization, even for lower-than-critical temperatures $T<T_{c}$, resulting in a "soft" conserved order parameter Ising model. Large fluctuations in the magnetization are suppressed in this case.

The dynamics of the system becomes more interesting once agents are allowed to follow two different strategies s.t. also the strategy $C_{i}(t)=-1$ occurs in the spin system which corresponds to a ferromagnetic coupling to the global magnetization. This strategy is called the chartist strategy as agents tend to follow the majority of traders. Initializing the model with a fixed ratio of the two strategies, one observes a transition between the conserved order parameter regime, where fundamentalists dominate, and a globally magnetized state with strongly attractive fixed points at the two points of maximum magnetization. This is where chartists dominate.

It remains to define the transition rule between the two strategies as each trader will tend to choose an optimal strategy which in general will not result in a fixed ratio of the two strategies. Let us consider the simplest possible scenario for local strategy changes. An agent who is in the majority group will often tend to switch to the minority group, e.g. in order to opt for future prospective returns of that not-yet fashionable commodity (and possibly escape a future crash of its currently popular good). As such an option is only available at a cost (the effort to obtain information, the potential risk as it affects prospective future return, etc.), the corresponding term will increase the local energy of the agent. Vice versa, let us assume that an agent who is in the minority (thus expecting future returns) might be unsatisfied with present returns, the more so, the larger the majority group of agents is, whose returns correlate with the current popularity of the commodity. Again, this option comes at a price accounted for by a positive energy term. The scenario is summarized under the simple assumption that a trader in the majority will always choose strategy $C_{i}(t)=1$, while a minority trader will always choose $C_{i}(t)=-1$ : Each trader chooses the risky strategy for the prospect of higher returns. The dynamics of the strategy spins then is

$$
C_{i}(t+1)=-C_{i}(t) \quad \text { if } \quad \alpha S_{i}(t) C_{i}(t) \sum_{j=1}^{N} S_{j}(t)<0
$$

Each agent thus faces a certain penalty for his strategic action, which increases with the absolute value of mag- 
netization. This is motivated by the fact that in general speculation costs rise in times with high volatility.

A particularly simple model is obtained, if the strategy adjustment is assumed to be done instantaneously. Then, the strategy spin drops out altogether and we obtain a simple spin model with the local field defined as

$$
h_{i}=\sum_{j=1}^{N} J_{i j} S_{j}-\alpha S_{i}\left|\frac{1}{N} \sum_{j=1}^{N} S_{j}\right|
$$

with a global coupling constant $\alpha>0$. While the first term tends to align the spin with its neighbors, the second term tends to encourage a spin flip when magnetization gets large.

The dynamics of this model is characterized by metastable phases of approximate undercritical Ising dynamics and intermediate phases of rapid rearrangements, reminiscent of overcritical dynamics. An example of subsequent snapshots in a $2 d$ system is shown in Fig. 1.

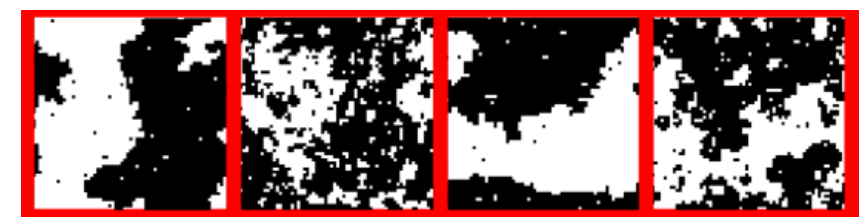

FIG. 1. Subsequent snapshots of the spin dynamics of a $32 \times 32$ lattice at undercritical temperature, taken at $t=611$, 2046, 2913, 3527 sweeps (Monte Carlo updates). The first and third snapshots are taken during a metastable phase, the second and fourth are in the turbulent regime, where memory of global spatial structures disintegrates.

Here, a 32x32 lattice of the general version of the model as defined in eq. (2) and where the strategy spins are updated according to eq. (3) is shown. It is simulated at temperature $T=1 / \beta=1.5$ and with couplings $J=1$ and $\alpha=4$, using random serial and asynchronous heat bath updates of single sites. Note that the temperature is below the critical temperature $T<T_{c}=2.269$ of the case $\alpha=0$. For every spin $S_{i}$ that is updated, the strategy spin $C_{i}$ is updated subsequently. The way the change of strategies is defined here, the model is to a large extent identical to the simplified version eq. (4), but in addition keeps track of the ratio of strategy choices. A metastable phase occurs at randomly frozen finite magnetization values, which, in the language of market dynamics, is the analogue of a bubble-related value of a good that emerged without any fundamental cause. Identifying a spin $S_{i}=+1$ with a buyer and $S_{i}=-1$ with a seller, the spin value can be viewed as the demand of an agent $i$ and the total excess demand, or magnetization, can be related to global price changes [7. Thus interpreting magnetization $M(t)=\frac{1}{N} \sum_{j=1}^{N} S_{j}$ as a measure of price, it is interesting to plot its logarithmic relative change $\operatorname{ret}(t)=\ln (M(t))-\ln (M(t-1))$, where intermittent phases are nicely visible (Fig. 2).

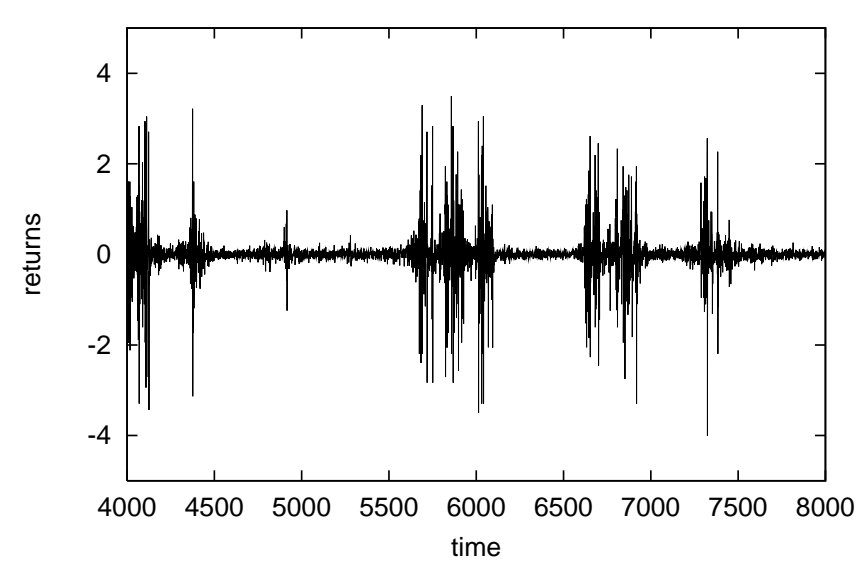

FIG. 2. Return or logarithmic relative change of magnetization of the $2 d$ model as defined above.

Comparing this observable to economic returns, features as non-Gaussian fluctuations and clustered phases of large fluctuations are observed here that are also known from real economic data. More specifically, the cumulative distribution of returns as derived from the magnetization of this model shows a pronounced powerlaw scaling (Fig. 3).

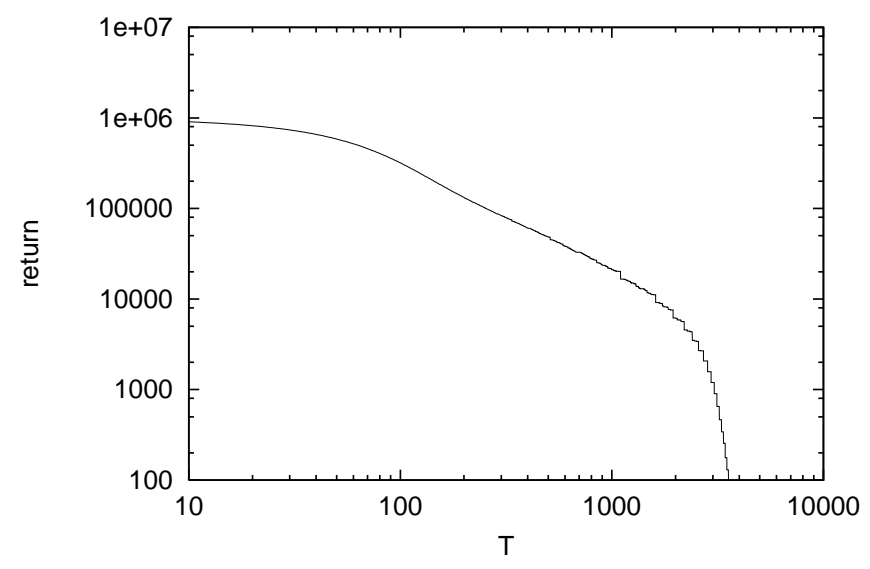

FIG. 3. Cumulative distribution of absolute returns exhibits power-law scaling. The $32 \times 32$ model is defined as above with parameters $T=1.0$ and $\alpha=8$ and sampled over $10^{6}$ sweeps.

The second feature, also seen in Fig. 2, are phases of high volatility that are strongly clustered. To quantify this, the corresponding autocorrelation of absolute returns is shown in Fig. 4. 


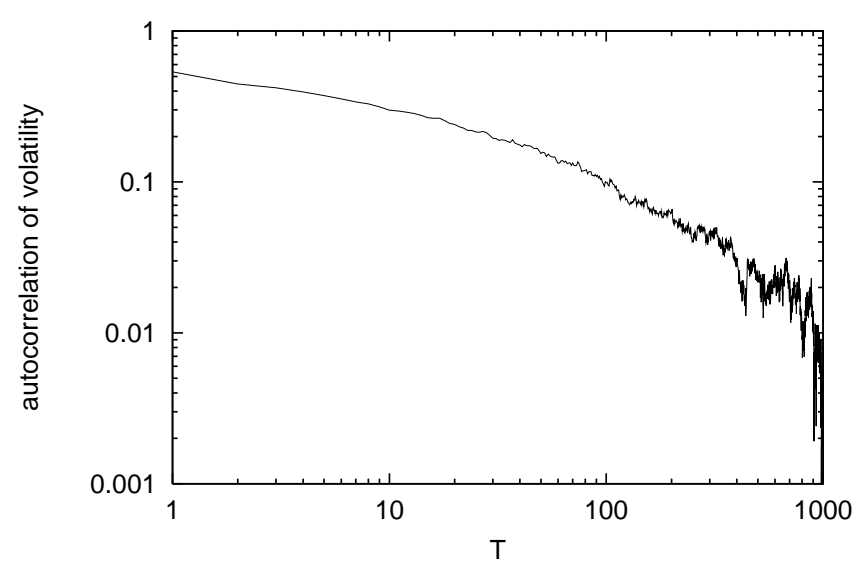

FIG. 4. Autocorrelation of absolute returns as a measure of volatility clustering. Model parameters are as in previous figure.

An interesting numerical experiment is to look at the first formulation of the above model, where strategy changes are explicitly tracked in the strategy spin variable $C_{i}(t)$ : Each agent switches its strategy to the opposite value, if the new strategy will be more risky (results in higher energy). This allows to see how strategy choices correlate to the phases of high volatility. Indeed, phases of high variance in changes of magnetization correlate with the number of agents that play the chartist strategy (Fig. 5).

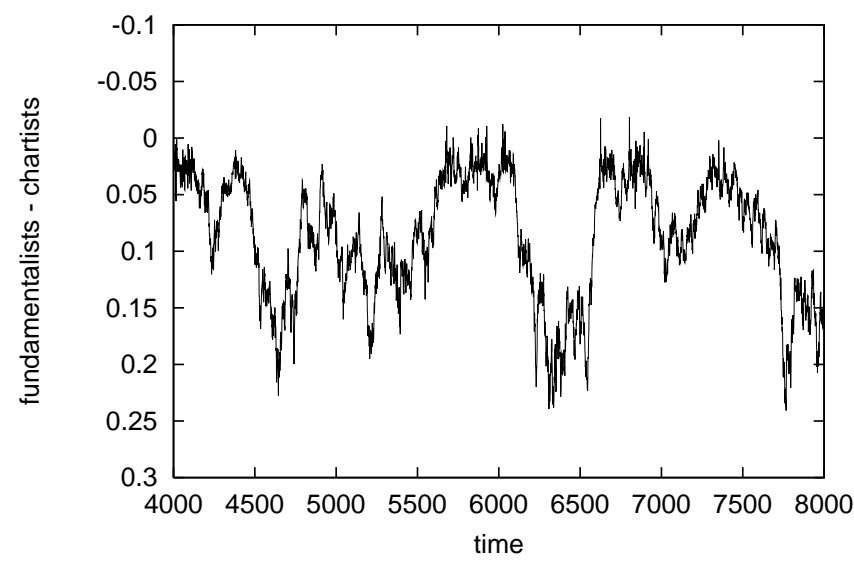

FIG. 5. Fraction of chartists in the same run as shown in Fig. 1. A high number of chartists coincides with phases of high volatility.

The simple spin model studied here reproduces major observational features of real economic markets. Due to its simplicity, it offers a chance to understand basic mechanisms at work in economic systems of many agents competing for commodities, as well as the possibility of analytic tractability that is often not given in complex agent simulations of the same phenomena.

We here considered the model in $2 d$ which is the lowest non-trivial number of dimensions. Many simple variants of the model are an obvious target of future research: Other dimensions or neighborhoods, the spin glass variants with random couplings, and other strategy changing rules (when including an explicit strategy spin), as well as a general $q$-state model with more than 2 states.

The spin model studied here shows intermittent behavior as a result of competition between conflicting local and global interactions. While exhibiting a phase transition at higher temperatures, it is considered here in the undercritical phase. As a result, the basic observation occurs in a wide temperature range and does not depend on tuning: Self-organization of the spin system to a regime dominated by metastable phases with intermittent disorder.

[1] G.J. Stigler, Public Regulation of the Securities Market, Journal of Business 37 (1964) 117-127.

[2] G.W. Kim and H.M. Markowitz, Investment rules, margin, and market volatility. J. Portfolio Management, 16, Fall 1989, 45-52.

[3] A. Kirman, The significance of testing for bubbles, mimeo: European Univ. Institute (1992); Ants, rationality, and recruitment, Quarterly J. Economics 108 (1993) 137-156.

[4] B.M. Roehner and D. Sornette, Thermometers of speculative frenzy, Eur. Phys. J. B 16 (2000) 729-739.

[5] M. Levy, H. Levy, and S. Solomon, A microscopic model of the stock market: Cycles, booms and crashes, Economics Letters 45 (1994) 103-111.

[6] M. Youssefmir and B. Huberman, Clustered volatility in multiagent dynamics, J. Economic Behavior and Organizsation 32 (1997) 101-118.

[7] R. Cont and J.-P. Bouchaud, Herd behavior and aggregate fluctuations in financial markets, Macroeconomic Dynamics 4 (2000) 170-196.

[8] W.B. Arthur, Inductive reasoning and bounded rationality, American Economic Review 84 (1994) 406-411.

[9] D. Challet and Y.C. Zhang, Emergence of cooperation and organization in an evolutionary game, Physica A 246 (1997) 498-500.

[10] D. Challet, M. Marsili, and Y.C. Zhang, Stylized facts of financial markets and market crashes in minority games, cond-mat/0101326.

[11] R.G. Palmer, W.B. Arthur, J.H. Holland, B. LeBaron, and P. Taylor, Artificial economic life: A simple model of a stockmarket, Physica D 75 (1994) 264-274.

[12] T. Lux and M. Marchesi, Scaling and criticality in a stochastic multi-agent model of a financial market, Nature 397 (1999) 498-500.

[13] T. Lux and M. Marchesi, Volatility clustering in financial markets: A microsimulation of interacting agents, Int. J. Theor. and Appl. Finance 3 (2000) 675-702.

[14] H. Foellmer, Random economies with many interacting agents, J. Math. Econ. 1 (1974) 51-62. 
[15] L.E. Blume, The statistical mechanics of strategic interaction, Games Econ. Behav. 5 (1993) 387-424.

[16] M. Aoki, New approaches to macroeconomic modeling, Cambridge University Press, 1996.

[17] T. Kaizoji, Speculative bubbles and crashes in stock markets: An interacting-agent model of speculative activity, cond-mat/0010263.

[18] P. Gopikrishnan, V. Plerou, L.A. Amaral, M. Meyer, and H.E. Stanley, Scaling of the distribution of fluctuations of financial market indices, Phys. Rev. E 60 (1999) 53055316.

[19] J.-P. Bouchaud, Power-laws in economy and finance: some ideas from physics, Quantitative Finance 1 (2001) 105.

[20] D. Chowdhury and D. Stauffer, A generalized spin model of financial markets, European Physical Journal B 8 (1999) 477-482.

[21] G. Iori, Avalanche dynamics and trading friction effects on stock market returns, Int. J. Mod. Phys. C 10 (1999) 1149-1162.

[22] Z.-F. Huang and S. Solomon, Finite market size as a source of extreme wealth inequality and market instability, Physica A 194 (2001) 503-513. 